\title{
Uso de la bola adiposa de Bichat como injerto para tratar la recesión gingival
}

\author{
Use of Bichat's adipose body as a graft to treat gingival recession
}

Britto Ebert Falcón-Guerrero « 1,a , Guido Sebastián Falcón-Pasapera 2,b

Filiación y grado académico

Universidad Latinoamericana CIMA. Tacna, Perú

Universidad Peruana Cayetano Heredia, Lima,

Perú.

a Doctor en Estomatología.

b Estudiante de pregrado.

D ORCID iD de Britto Falcón-Guerrero https://orcid.org/0000-0002-9585-7052

10 ORCID ID de Guido Sebastián Falcón https://orcid.org/0000-0003-3433-1599

Fuentes de financiamiento

Autofinanciado.

Conflictos de interés

Los autores declaran no tener conflictos de interés en la publicación de este artículo.

Recibido: 05-04-2021

Arbitrados por pares

Aceptado: 13-07-2021

Citar como

Falcón-Guerrero BE, Falcón-Pasapera GS. Uso de la bola adiposa de Bichat como injerto para tratar la recesión gingival. Rev Peru Cienc Salud. 2021 3(3): 195-201. doi: https://doi.org/10.37711/ rpcs.2021.3.3.338

Correspondencia

\section{RESUMEN}

Objetivo. Recopilar la evidencia científica sobre los resultados clínicos que se obtienen al usar la bola adiposa de Bichat (BAB) en el tratamiento de las recesiones gingivales. Métodos. Se revisaron las bases de datos online PubMed, Cochrane y Wiley Online Library. Utilizando los siguientes términos de búsqueda: "buccal" AND "adipose tissue" OR "fat pad" AND "coverage" AND "gingival recession". Al combinar las búsquedas y filtrar solo los documentos que contengan el tema objetivo de la revisión en idioma inglés, se generaron solo 9 artículos que cumplen con los criterios de inclusión. Resultados. Se analizó la información encontrada donde fueron usados injertos pediculados y libres con la bola adiposa de Bichat, encontrando buenos resultados en todos los casos; lo que significa que esta es una buena alternativa autóloga para el tratamiento de las recesiones gingivales, ante las limitaciones de los injertos del paladar. Conclusiones. El uso de la bola adiposa de Bichat es una propuesta autóloga prometedora y representa una buena alternativa para el éxito del tratamiento de las recesiones gingivales.

Palabras clave: autoinjertos; trasplante autólogo; cirugía bucal; dental; estética; recesión gingival; tejido adiposo (fuente: DeCS - BIREME).

\section{ABSTRACT}

Objective. Review was to gather scientific evidence on the clinical results obtained by using the Bichat's adipose body (BAB) in the treatment of gingival recessions. Methods. The PubMed, Cochrane and Wiley Online Library databases were reviewed. Using the following search terms: "buccal" AND "adipose tissue" OR "fat pad" AND "coverage" AND "gingival recession". By combining searches and filtering only documents that contain the Englishlanguage review target, only 9 articles that meet the inclusion criteria were generated. Results. The information found, where pediculated and free grafts with the Bichat's adipose body were used, was analyzed, finding good results in all cases; which means that this is a good autologous alternative for the treatment of gingival recessions, in the face of the limitations of palate grafts. Conclusions. The use of Bichat's adipose body is a promising autologous proposal and represents a good alternative for the success of the treatment of gingival recessions.

Keywords: autografts; autologous transplant; oral surgery; dentistry; aesthetic; gingival recession; adipose tissue (source: MeSH-NLM). 


\section{INTRODUCCIÓN}

La recesión gingival es el desplazamiento del margen gingival apical a la unión cemento-esmalte, provocando la exposición radicular ${ }^{(1)}$; lo que origina complicaciones como la hipersensibilidad dental, molestias estéticas y susceptibilidad a la caries radicular (2). Para el manejo de estas complicaciones se usa el colgajo de reposición coronal, injerto gingival libre y de tejido conectivo; sin embargo, estas alternativas se asocian con molestias postoperatorias, necrosis tisular y sangrado, debido a que se puede lesionar la arteria palatina mayor ${ }^{(3)}$.

El uso del injerto de tejido conectivo asociado con el colgajo de reposición coronal es considerado el estándar de oro para la cobertura radicular; lo que mejora el nivel de inserción clínica, se gana mayor cantidad de tejido queratinizado, reduce la bolsa periodontal y, se recupera el color y la textura del tejido ${ }^{(4)}$. También existen otras alternativas que no requieren de una segunda cirugía, como los factores de crecimiento derivados de plaquetas (PDGF), fibrina rica en plaquetas (PRF) y los derivados de la matriz del esmalte (EMD), que ayudan a mantener la cobertura radicular de forma estable entre 5 a 10 años ${ }^{(5,6)}$.

Sin embargo, el tratamiento de recesiones múltiples de tres o más dientes está directamente relacionado con la cantidad de tejido conectivo disponible, ya que si no es suficiente para cubrir las recesiones, se requerirá más de un procedimiento quirúrgico. Por lo que, está limitado por la cantidad y calidad de tejido blando disponible y la dificultad anatómica del área donante ${ }^{(1,7)}$. Han surgido alternativas para poder suplir la falta de tejido conectivo, como los derivados del porcino: la matriz a base de colágeno (Mucograft ${ }^{\oplus}$ ) y la matriz dérmica acelular, que brindan buenos resultados histológicos, con integración exitosa y ausencia de eventos adversos; mostrando un tejido maduro presente en el área quirúrgica donde se usó estos biomateriales ${ }^{(8,9)}$.

Del mismo modo, se ha usado la membrana placentaria amniótica (corion/amnios) con colgajo de reposición coronal para las recesiones gingivales múltiples, brindando una opción efectiva de tratamiento para recesiones de clase I y II ${ }^{(10,11)}$. Sin embargo, estas alternativas implican una mayor inversión y costos del tratamiento de nuestros pacientes; por lo que puede ser inviable en muchas situaciones clínicas.

El Haddad et al. ${ }^{(12)}$ presentan en el año 2008 una nueva técnica para la cobertura de la raíz con defectos graves de recesión gingival, utilizando la bola adiposa de Bichat (BAB) pediculada, que proporciona una nueva fuente de suficiente tejido con buen suministro sanguíneo, de lo que obtienen una cantidad clínicamente significativa de encía queratinizada que cubría el defecto de recesión de la raíz. Esta es, por consiguiente, una aplicación novedosa con resultados prometedores para la cobertura de la raíz.

Considerando que es necesario contar con nuevas alternativas autólogas que suplan al paladar como zona donante de tejido blando, es que la presente revisión tuvo como objetivo analizar la evidencia científica sobre el uso de la BAB o almohadilla de grasa bucal en el tratamiento de las recesiones gingivales.

\section{MÉTODOS}

La estrategia de búsqueda desarrollada para la revisión se llevó a cabo en las bases de datos online PubMed, Cochrane y en Wiley Online Library, mediante términos de indización específicos para obtener información de todos los casos clínicos, revisiones y estudios originales sobre el uso de la BAB o almohadilla de grasa bucal en el tratamiento de las recesiones gingivales. Fueron utilizados los siguientes términos de búsqueda: "buccal" [All Fields] AND ("adipose tissue"[MeSH Terms] OR ("adipose"[All Fields] AND "tissue"[All Fields]) OR "adipose tissue"[All Fields] OR ("fat"[All Fields] AND "pad"[All Fields]) OR "fat pad"[All Fields]) AND ("plant roots"[MeSH Terms] OR ("plant"[All Fields] AND "roots"[All Fields]) OR "plant roots"[All Fields] OR "root"[All Fields]) AND ("AHIP Cover"[Journal] OR "coverage"[All Fields]) AND ("gingival recession"[MeSH Terms] OR ("gingival"[All Fields] AND "recession"[All Fields]) OR "gingival recession"[All Fields]): "2020/07/09"[PDat]).

Al combinar las búsquedas y filtrar solo los documentos que contengan el tema objetivo de la revisión en idioma inglés, se generaron 21 artículos de los cuales ocho eran duplicados y uno solo tenía el abstract y no se pudo acceder al texto completo, quedando 12 artículos; de estos, uno era una revisión bibliográfica que no tiene como objetivo principal la cobertura radicular con BAB y dos están referidos a los implantes dentales. Por lo tanto, quedan finalmente solo 9 artículos para ser utilizados en el presente trabajo. Esta muestra estuvo constituida de dos estudios clínicos, un estudio retrospectivo, una serie de casos y cinco reportes de casos (ver Figura 1).

\section{RESULTADOS}

\section{Bola adiposa de bichat (BAB) o bolsa de grasa bucal}

La BAB fue descrita por primera vez en 1732 por Heister, pero debe su nombre a la descripción realizada en 1802 por Bichat (citado por Bravo et al. ${ }^{(13)}$. Consiste en una masa lobulada llamada cuerpo central, conformado por 


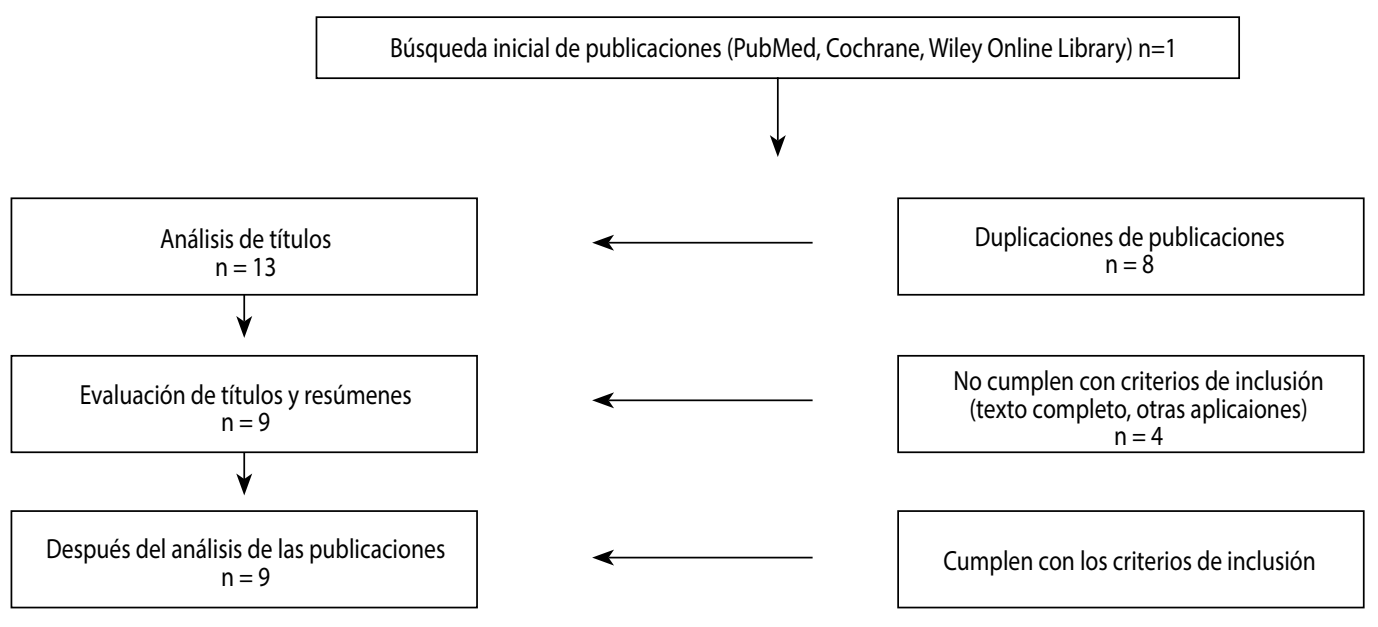

Figura 1. Diagrama de la revisión donde se identifican nueve publicaciones incluidas para su análisis

tres lóbulos: anterior, intermedio y posterior. El lóbulo posterior tiene cuatro extensiones digitiformes: bucal, pterigoidea, pterigopalatina y temporal, las cuales se extienden desde el centro hacia los espacios adyacentes. El cuerpo central se ubica en el espacio masticatorio, entre el músculo buccinador y el borde anterior del músculo masetero, cubierto por una fina cápsula de fascia; yace superior al conducto parotídeo, se extiende hacia el maxilar por posterior y hacia anterior por el vestí-bulo bucal. Los vasos faciales marcan el límite anterior.

La extensión bucal de la BAB yace superficialmente en la mejilla, sobre la fascia bucofaríngea que delimita la superficie externa del músculo buccinador, ayudando a formar el contorno facial. La extensión bucal y el cuerpo central constituyen entre el 55 a $70 \%$ del peso total. La extensión temporal se extiende bajo el arco cigomático hacia el plano temporal donde se divide en dos porciones: una porción superficial, más grande, la cual se extiende hacia la superior, entre la fascia temporal y la superficie del músculo temporal; y una porción profunda que es más delgada y pasa entre las fibras superficiales y profundas del músculo temporal hasta llegar al espacio temporal.

La extensión temporal es el único proceso que no puede ser fácilmente separado de sus tejidos adyacentes. La extensión pterigoidea es una prolongación posterior que generalmente se encuentra en el espacio pterigomandibular, rodeando a los paquetes neurovasculares y al nervio lingual. Cada proceso tiene su propia cápsula y se ancla a estructuras cercanas mediante ligamentos. Histológicamente, la BAB es diferente al tejido adiposo subcutáneo y está compuesta por el mismo tipo de grasa que la grasa orbitaria. La BAB contiene células madre mesenquimales pluripotentes que caracterizan por una tasa de mitosis más alta que las células pluripotentes aisladas, teniendo un gran potencial regenerativo ${ }^{(13,14)}$.

El tamaño de la $B A B$ es estructuralmente el mismo, entre diferentes personas, independientemente del peso corporal general y la distribución de grasa. Su volumen es de aproximadamente $10 \mathrm{ml}$ con un tamaño de aproximadamente $60 \mathrm{~mm}$ de largo, $50 \mathrm{~mm}$ de ancho y $6 \mathrm{~mm}$ de espesor. Otra característica es la distribución de los vasos sanguíneos representada por los vasos del maxilar (bucal y temporal profundo), la arteria temporal superficial (rama facial transversal) y las arterias faciales (ramas pequeñas) ${ }^{(14)}$.

Esta estructura anatómica es necesaria ya que cumple diversas funciones, tales como separar los músculos masticatorios entre sí para mejorar su movilidad, rellenar espacios masticatorios y faciales, contrarrestar la presión negativa generada por la succión en los recién nacidos, protección para complejos neurovasculares, actuar como red venosa asociada al plexo pterigoideo, entre otras ${ }^{(13)}$.

La BAB se utiliza para numerosas aplicaciones, como el tratamiento de enfermedades congénitas oroantrales u oronasales, reparación congénita del paladar hendido, defecto maligno intraoral, la reparación de defectos quirúrgicos después de la escisión de leucoplasia y fibrosis submucosa, la cobertura de injertos de hueso maxilar y mandibular, y el revestimiento de la superficie de los injertos de hueso del seno maxilar en el procedimiento de elevación de seno, artroplastia de la articulación 
temporomandibular (ATM), alargamiento del paladar blando, aumento de las cuerdas vocales, osteonecrosis de la mandíbula, adenoma pleomórfico y cobertura de la raíz ${ }^{(15-18)}$.

Por su parte, Porcaro et al. ${ }^{(19)}$ proponen el uso de la $B A B$ en el caso de la osteorradionecrosis, que es una complicación potencialmente debilitante de la radioterapia en pacientes con cánceres de cabeza y cuello. Esta alternativa asegura el cierre primario, brinda protección adecuada y un suministro considerable de sangre durante la etapa inicial de curación; además, sirve como un reservorio de células madre derivadas de tejido adiposo que pueden formar osteoides y diferenciarse en múltiples linajes mesodérmicos.

El primero en usar la BAB para injerto de grasa fue Egyedy en 1977 (citado por Bravo et al. ${ }^{(13)}$ ) para cerrar una comunicación oroantral, quien usón un colgajo pediculado que ha demostrado ser exitoso y tiene varias ventajas: su ubicación permite su fácil uso, mínima disección, tiene gran versatilidad, buena movilidad, gran aporte sanguíneo, baja tasa de complicaciones, escasa morbilidad del sitio donante, bajo riesgo de infección, rápida técnica quirúrgica, rápida epitelización, sin cicatriz visible, entre otras ${ }^{(13)}$. Es así que Alonso-González et al. ${ }^{(20)}$ evaluaron el cierre de las comunicaciones oroantrales y el nivel de satisfacción del paciente con la $B A B$, encontrando que los pacientes estaban muy satisfechos en general con el tratamiento y con la fonética, la estética y la masticación.

Lograr un periodo posquirúrgico con menos complicaciones es una condición de éxito, que determina una recuperación más cómoda para el paciente. Por lo que ahora se recomienda usar la fotobiomodulación con láser de baja potencia como alternativa efectiva para el control del edema, el dolor, la reducción de la inflamación y la promoción de la comodidad del paciente en la cirugía postbichectomía ${ }^{(21)}$. Sin embargo, es necesario tener en cuenta que hay un aumento de lesiones y secuelas postoperatorias al realizar este procedimiento quirúrgico que provoca lesiones de la glándula parótida y la arteria bucal, lo que se confirmó mediante resonancia magnética nuclear $^{(22)}$.

\section{Uso de la bola adiposa de Bichat (BAB) en las recesiones radiculares}

Los estudios hallados en los motores de búsqueda son limitados, por lo que todos estos se analizan y sus características se detallan en la tabla 1.

Después del análisis se encontró un total de 58 casos tratados, los cuales presentaron recesiones Miller clase I, II, III y IV. La BAB se usó tanto como injerto pediculado y como injerto libre, obteniéndose coberturas radiculares totales y parciales de las recesiones tratadas; lo cual representa que este procedimiento es una buena alternativa de tratamiento.

El uso de la $B A B$ para las recesiones gingivales fue presentado el año 2008, por El Haddad et al. ${ }^{(12)}$, quienes realizan la cobertura de la raíz en una recesión gingival grave usando la BAB pediculada, obteniendo una cantidad clínicamente significativa de encía queratinizada, con un $100 \%$ de cubertura a pesar de movilidad en la molar.

Agarwal et al. ${ }^{(23)}$ mencionan que las células grasas se reemplazan por completo con tejido fibroso relativamente celular y se cubren con epitelio escamoso estratificado, observando epitelización de la BAB pediculada después

Tabla 1. Características del uso de la $B A B$ en los artículos analizados

\begin{tabular}{|c|c|c|c|c|c|}
\hline Autor - año & Tipo de estudio & Técnica & Recesión & Muestra & Cobertura radicular \\
\hline El Haddad et al., $2008^{(12)}$ & Reporte de caso & Pediculado & Miller IV & 1 & Total \\
\hline Agarwal et al., $20144^{(23)}$ & Reporte de caso & Pediculado & Miller IV & 1 & Parcial \\
\hline Deliberador et al., $2015^{(24)}$ & $\begin{array}{c}\text { Estudio clínico controlado a } \\
\text { boca partida }\end{array}$ & Libre & Miller I y II & 12 & $\begin{array}{c}\text { Total-6 } \\
\text { Parcial-6 }\end{array}$ \\
\hline Kablan FK, $2018^{(25)}$ & Estudio retrospectivo & Libre & Miller II y III & 17 & $\begin{array}{l}\text { Total } \\
\text { Parcial }\end{array}$ \\
\hline Storrer et al., $2018^{(26)}$ & Reporte de caso & Libre & Miller I & 1 & $\begin{array}{l}\text { Total } \\
\text { Parcial }\end{array}$ \\
\hline Panda et al., $2016^{(27)}$ & Reporte de caso & Pediculado & Miller III & 3 & Parcial \\
\hline El-Haddad et al., $2017^{(28)}$ & Serie de casos & Pediculado & Miller IV & 10 & Total-4 \\
\hline Deepa et al., $2018^{(29)}$ & Estudio clínico & Pediculado & Miller II y III & 10 & Parcial-6 \\
\hline Grover et al., $2018^{(30)}$ & Reporte de caso & Pediculado & Miller II & 3 & Parcial \\
\hline
\end{tabular}


de la segunda semana y una epitelización completa entre 4-6 semanas. El colgajo se vuelve fácilmente accesible a través de una sola incisión, logrando una ganancia de inserción clínica en las recesiones de $4 \mathrm{~mm}$.

Deliberador et al. ${ }^{(24)}$ realizaron un estudio clínico comparativo a boca partida entre el injerto de la BAB no pediculada y el injerto libre de tejido conectivo para el tratamiento de recesiones gingivales, indicando que el uso del injerto de BAB tiene resultados clínicos similares al del tejido subepitelial y ambos se consideran como métodos exitosos para tratar las recesiones gingivales Miller Clase I y II; encontrando una media de 67,50 \pm 47,21 para el grupo experimental. El ancho del tejido queratinizado no difirió significativamente entre los grupos a los 6 meses de seguimiento.

De manera similar, Kablan et al. ${ }^{(25)}$ utilizaron la BAB como un injerto libre para cubrir las raíces expuestas en dientes maxilares anteriores y en los dientes mandibulares. Al recolectar injertos libres de ambas $B A B$, se puede proporcionar suficiente volumen para tratar múltiples recesiones gingivales y así tratar la boca completa, y que sepamos, no hay un sitio donante de tejido conectivo en la cavidad oral que pueda proporcionar la misma cantidad de injerto. En un seguimiento a los 12 meses, se produjo una reducción significativa de la recesión, de 4,8 $\pm 1,8 \mathrm{~mm}$ a $1,7 \pm 0,9 \mathrm{~mm}$; logrando una reducción media de 3,1 mm en comparación con el valor inicial. Concluyendo entonces que la estabilidad a largo plazo de la cobertura obtenida de los dientes expuestos la convierte en una buena y excelente opción para el tratamiento de recesiones gingivales en ambos maxilares.

Recientemente, Storrer et al. ${ }^{(26)}$ presentaron un injerto libre de $B A B$ con un seguimiento de 365 días, en un caso de recesión Miller Clase I, logrando una cubertura estable del $100 \%$ e indicando, por consiguiente, que es una técnica eficaz, segura y fácil de realizar, y puede considerarse una opción de tratamiento.

Panda et al. ${ }^{(27)}$ mencionan que la BAB, utilizada como un injerto subepitelial, aumenta la previsibilidad y el resultado de los procedimientos de cobertura de raíz en casos con mal pronóstico, debido a su menor morbilidad del sitio donante, vascularización y resultado favorable.

El Haddad et al. (28) evalúan en su estudio el efecto a largo plazo de la BAB pediculada sola y con Emdogain. A los 2 años, el porcentaje de cobertura de la raíz fue de $60,18 \%$ y $59,07 \%$ para el grupo I y el grupo II, respectivamente. El Emdogain con $B A B$ no mostró una mejora significativa en comparación con la BAB sola, en términos de porcentaje de cobertura de raíz. De lo que se concluye que la $B A B$ pediculada sola puede ser el mejor tratamiento para defectos graves de recesión gingival, ya que mantiene un excelente suministro de sangre para los tejidos del injerto y así consigue evitar una posible necrosis y una falla en la cobertura de la raíz.

Del mismo modo, Deepa et al. ${ }^{(29)}$ evaluaron el uso de la $B A B$ pediculada para el tratamiento de la recesión gingival clase II y III, encontrando un 89,3\% de cobertura de la raíz, con una disminución estadísticamente significativa en el ancho y la profundidad de la recesión; observando una epitelización completa entre 4 y 6 semanas; de lo que se desprende que esta es una alternativa útil en el tratamiento de la recesión gingival severa.

Grover et al. (30) aplicaron la BAB en recesiones gingivales clase II de $5 \mathrm{~mm}$, que tenían abrasiones, las que se restauraron con cemento de ionómero de vidrio; hallando que a los tres años del postoperatorio, el margen gingival estaba en la unión cemento-esmalte, estable y saludable y sin ninguna recesión.

También, se ha descrito que estos tejidos adiposos son una fuente prometedora de células mesenquimales que pueden obtenerse con una molestia mínima para los pacientes; es así, que la BAB es una fuente de células madre fácilmente accesible que se puede obtener de forma intraoral. La comparación de células madre adiposas derivadas de la $\mathrm{BAB}$, con tejidos adiposos de otras partes del cuerpo, indican un rendimiento celular, morfología y diferenciación multilínea similar. Sin embargo, las células madre derivadas de la $B A B$ proliferan más rápido y son más propensas a producir colonias que los tejidos adiposos de otras partes del cuerpo ${ }^{(31)}$. Esto demuestra que existe una diferenciación para producir osteoblastos, lo que confirma la presencia de células madre dentro de la BAB que pueden ayudar en la regeneración periodontal (28).

Por otro lado, Kablan ${ }^{(32)}$ informó una serie de casos que describían el uso de la BAB más injertos óseos para el tratamiento de la periimplantitis, con un período medio de seguimiento de 12 meses; mejorando el nivel de inserción clínica de los tejidos blandos alrededor de los implantes afectados, alargando la supervivencia del implante dental con excelentes resultados funcionales y estéticos e inhibiendo la recurrencia de las lesiones periimplantarias. De manera similar, Guennal et al. ${ }^{(33)}$ reportan el uso de la $B A B$ en implantes cigomáticos, como un injerto libre para cubrir los implantes, lo que proporciona una encía y mucosa más gruesa en la cara vestibular. Así mismo, de veinticinco pacientes, ninguno tuvo una recesión gingival durante el seguimiento, lo 
que permite retener una cantidad considerable de encía gruesa alrededor de los implantes y evitar la exposición a largo plazo de los implantes en la cara vestibular.

\section{Ventajas}

Ubicación anatómica favorable; la proximidad y accesibilidad del sitio donante a la cavidad oral; la morbilidad del sitio donante y el malestar del paciente son mínimos; la facilidad y mínima disección con la que se puede cosechar y movilizar el injerto; excelente suministro de sangre; simplicidad; versatilidad; las irregularidades superficiales del tejido queratinizado formado se pueden remodelar completa y fácilmente con gingivoplastia ${ }^{(12,23)}$; esta puede usarse de tipo pediculado y no pediculado (injerto libre) ${ }^{(24)}$; volumen adecuado y notable resistencia a la infección; ${ }^{(29)}$ proporciona una gran cantidad de tejido ${ }^{(25)}$ y una excelente combinación en el resultado del color y la textura ${ }^{(30)}$.

\section{Desventajas o limitaciones}

Estiramiento limitado del colgajo pediculado para preservar su vascularización; no podría usarse en la parte mandibular como un colgajo pediculado ${ }^{(12,23)}$.

También se ha reportado sangrado, hematoma, necrosis parcial, cicatrización excesiva e infección ${ }^{(29)}$. Está contraindicada en dientes móviles con pérdida ósea extensa, pacientes que no cooperan y que no están dispuestos a mantener una buena higiene oral, y en estados de infección o presencia de tumoraciones en el sitio de la $B A B{ }^{(30)}$.

\section{Conclusiones}

En relación a la recopilación de la literatura científica efectuada, se destaca que el uso de la BAB está asociado con el éxito del tratamiento de las recesiones gingivales, siendo un procedimiento quirúrgico simple, seguro y efectivo que puede ser usado tanto de manera pediculada como de injerto libre, disponiendo de una gran cantidad de tejido para ser usado. De todos modos y ante la escasa información existente, es necesario contar con más estudios que tengan un mayor tiempo de seguimiento para indicar la fiabilidad a largo plazo del uso de la BAB en el tratamiento de las recesiones gingivales.

\section{REFERENCIAS}

1. Novaes $A B$ Jr., Palioto DB. Experimental and clinical studies on plastic periodontal procedures. Periodontol 2000 [Internet]. 2019 [Consultado 2020 Jul 09];79(1):5680. doi: $10.1111 /$ prd.12247

2. Carranza N, Rojas MA. Treatment of an Advanced Gingival Recession Involving the Apex of the Tooth: Periodontal Plastic, Endodontic Surgical Approach With a La- terally Stretched Flap and a Connective Tissue Graft. Clin Adv Periodontics. [Internet] 2019 [Consultado 2020 Jul 09];9(2):70-76. doi: 10.10012/cap.10054

3. Al-Shibani N. Low-intensity laser for harvesting palatal graft for the treatment of gingival recession: $A$ systematic review. J Investig Clin Dent. [Internet] 2019 [Consultado 2020 Jul 09];10(1):e12368. doi: 10.1111/ jicd. 12368

4. Moraschini V, de Almeida DCF, Sartoretto S, Bailly Guimarães $\mathrm{H}$, Chaves Cavalcante I, Diuana Calasans-Maia $M$. Clinical efficacy of xenogeneic collagen matrix in the treatment of gingival recession: a systematic review and meta-analysis. Acta Odontol Scand. [Internet] 2019 [Consultado $2020 \mathrm{Jul}$ 09];77(6):457-467. doi: 10.1080/00016357.2019.1588372

5. Barootchi S, Tavelli L, Di Gianfilippo R, Byun HY, Oh TJ, Barbato $L$, et al. Long term assessment of root coverage stability using connective tissue graft with or without an epithelial collar for gingival recession treatment. A 12-year follow-up from a randomized clinical trial. J Clin Periodontol. [Internet] 2019 [Consultado 2020 Jul 09];46(11):1124-1133. doi: 10.1111/jcpe.13187

6. Krishnakumar D, Mahendra J, Ari G, Perumalsamy R. A clinical and histological evaluation of platelet-rich fibrin and CGF for root coverage procedure using coronally advanced flap: A split-mouth design. Indian J Dent Res. [Internet] 2019 [Consultado 2020 Jul 09];30(6):970-974. https://doi.org/10.1111/jcpe.13187

7. Rotundo R, Genzano L, Patel D, D'Aiuto F, Nieri M. Adjunctive benefit of a xenogenic collagen matrix associated with coronally advanced flap for the treatment of multiple gingival recessions: A superiority, assessor-blind, randomized clinical trial. J Clin Periodontol. [Internet] 2019 [Consultado 2020 Jul 09];46(10):1013-1023. doi: $10.1111 /$ jcpe. 13168

8. Suárez-López F, Rodriguez JC, Asa'ad F, Wang HL. Comparison of two soft tissue substitutes for the treatment of gingival recession defects: an animal histological study. J Appl Oral Sci. [Internet] 2019 [Consultado 2020 Jul 09]; 27:e20180584. doi: 10.1590/1678-7757-2018-0584

9. Menceva Z, Dimitrovski O, Popovska M, Spasovski S, Spirov V, Petrushevska G. Free Gingival Graft versus Mucograft: Histological Evaluation. Open Access Maced J Med Sci. [Internet] 2018 [Consultado 2020 Jul 09];6(4):675679. doi: 10.3889/oamjms.2018.127

10. Bolla V, Reddy PK, Kalakonda B, Koppolu P, Manaswini E. Coronally Advanced Flap with Amniotic Membrane in the Treatment of Gingival Recession: Three Case Reports. Int J Appl Basic Med Res. [Internet] 2019 [Consultado 2020 Jul 09];9(2):111-114. doi: 10.4103/ijabmr. IJABMR_290_18

11. Chopra P, Kassal J, Masamatti SS, Grover HS. Comparative evaluation of clinical efficacy of coronally advanced flap alone and in combination with placental membrane and demineralized freeze-dried bone allograft in the treatment of gingival recession. J Indian Soc Periodontol. [Internet] 2019 [Consultado $2020 \mathrm{Jul}$ 09];23(2):137144. doi: 10.4103/JISP.JISP_308_18

12. El Haddad SA, Abd El Razzak MY, El Shall M. Use of pedicled buccal fat pad in root coverage of severe gingival recession defect. J Periodontol. [Internet] 2008 [Consultado 2020 Jul 09];79(7):1271-1279. doi: 10.1902/ jop.2008.070176 
13. Bravo G, Minzer Ferrer S, Fernández L. Sinusitis odontogénica, fístula oroantral y su reparación quirúrgica mediante colgajo de bolsa de Bichat: revisión de la literatura. Acta Otorrinolaringol Esp. [Internet] 2015 [Consultado 2020 Jul 09]; 67(2):107-113. doi: 10.1016/j.otorri.2015.03.001

14. Conti G, Bertossi D, Dai Prè E, Cavallini Ch, Scupoli MT, Ricciardi G, et al. Regenerative potential of the Bichat fat pad determined by the quantification of multilineage differentiating stress enduring cells. Eur J Histochem. [Internet] 2018 [Consultado 2020 Jul 09];62(4):2900. doi: 10.4081/ejh.2018.2900

15. Galletti C, Cammaroto G, Galletti F, Camps-Font O, Gay-Escoda C, Bara-Casaus JJ. Dental implants after the use of bichat's buccal fat pad for the sealing of oro-antral communications. A case report and literature review. J Clin Exp Dent. [Internet] 2016 [Consultado 2020 Jul 09];8(5):e645-e649. doi: 10.4317/jced.53318

16. Mannelli G, Arcuri F, Comini LV, Valente D, Spinelli G. Buccal Fat Pad: Report of 24 Cases and Literature Review of 1,635 Cases of Oral Defect Reconstruction. ORL J Otorhinolaryngol Relat Spec. [Internet] 2019 [Consultado 2020 Jul 09];81(1):24-35. doi: 10.1159/000494027

17. Oliveira JQ, Cetira Filho EL, Andrade GS, Silveira DXD, Carvalho ACGS. Technique of the Buccal Fat Pad Flap as an Alternative for the Surgical Defect of Pleomorphic Adenoma. J Craniofac Surg. [Internet] 2019 [Consultado 2020 Jul 09];30(3):798-799. doi: 10.1097/ SCS.0000000000004890

18. Peñarrocha-Oltra D, Alonso-González R, Pellicer-Chover $\mathrm{H}$, Aloy-Prósper A, Peñarrocha-Diago M. Closure of oroantral communication with buccal fat pad after removing bilateral failed zygomatic implants: A case report and 6-month follow-up. J Clin Exp Dent. [Internet] 2015 [Consultado $2020 \mathrm{Jul}$ 09];7(1):e159-e162. doi: 10.4317/jced.51741

19. Porcaro G, Amosso E, Mirabelli L, Busa A, Carini F, Maddalone M. Osteoradionecrosis of the Posterior Maxilla: A New Approach Combining Erbium: Yttrium Aluminium Garnet Laser and Bichat Bulla Flap. J Craniofac Surg. [Internet] 2015 [Consultado 2020 Jul 09];26(7):e627-e629. doi: 10.1097/SCS.0000000000002136

20. Alonso-González R, Peñarrocha-Diago MA, Peñarrocha-Oltra D, Aloy- Prósper A, Camacho-Alonso F, Peñarrocha-Diago $M$. Closure of oroantral communications with Bichat's buccal fat pad. Level of patient satisfaction. J Clin Exp Dent. [Internet] 2015 [Consultado 2020 Jul 09];7(1):e28-33. doi: 10.4317/jced.51730

21. Bernal CG, Kraul LF, Cardoso TW, Eduardo CP, Aranha ACC, de Freitas PM. Photobiomodulation in the Postoperative of Bichectomy Surgeries: Case Series. Photomed Laser Surg. [Internet] 2018 [Consultado 2020 Jul 09];36(7):391-394. doi: 10.1089/pho.2017.4407.

22. Vieira GM, Jorge FD, Franco EJ, Dias LDC, Guimarães MDCM, Oliveira LA. Lesions of the Parotid Gland and Buccal Artery After Buccal Fat Pad Reduction. J Craniofac
Surg. [Internet] 2019 [Consultado 2020 Jul 09];30(3):790792. doi: 10.1097/SCS.0000000000004880

23. Agarwal C, Gayathri GV, Mehta DS. An innovative technique for root coverage using pedicled buccal fat pad. Contemp Clin Dent. [Internet] 2014 [Consultado 2020 Jul 09];5(3):386-388. doi: 10.1111/jicd.12368

24. Deliberador TM, Trevisani CT, Storrer CL, et al. Non-Pedicled Buccal Fat Pad Grafts to Treatment for Class I and II Gingival Recessions: A Clinical Trial. Braz Dent J. [Internet] 2015 [Consultado $2020 \mathrm{Jul}$ 09];26(6):572-579. https://doi.org/10.1590/0103-6440201300432

25. Kablan FK. The Reliability of Free Buccal Fat Graft for Treatment of Severe Gingival Recessions at Mandibular and Maxillary Exposed Roots. Ann Maxillofac Surg. [Internet] 2018 [Consultado 2020 Jul 09];8(2):281-286. doi: 10.4103/ams.ams_149_18

26. Storrer CLM, Muller LL, Pissaia JF, Andrade CF, Trevisani CRT, Deliberador TM. Treatment of Miller Class I Gingival Recession with Using Nonpedicle Adipose Tissue after Bichectomy Surgical Technique: A Case Report. Case Rep Dent. [Internet] 2019 [Consultado 2020 Jul 09];2019:1049453. doi: 10.1111/jicd.12368

27. Panda S, Del Fabbro M, Satpathy A, Das AC. Pedicled buccal fat pad graft for root coverage in severe gingival recession defect. J Indian Soc Periodontol. [Internet] 2016 [Consultado 2020 Jul 09];20(2):216-219. doi: 10.4103/0972-124X.170844

28. El-Haddad SA, El-Shall MA. Two-Year Clinical Evaluation of Pedicled Buccal Fat Pad Alone and with Emdogain for Root Coverage of Severe Gingival Recession Defects: A Case Series. Int J Periodontics Restorative Dent. [Internet] 2017 [Consultado $2020 \mathrm{Jul}$ 09];37(6):901-907. doi: $10.11607 /$ prd. 2630

29. Deepa D, Arun Kumar KV. Clinical evaluation of Class II and Class III gingival recession defects of maxillary posterior teeth treated with pedicled buccal fat pad: A pilot study. Dent Res J (Isfahan). [Internet] 2018 [Consultado 2020 Jul 09];15(1):11-16. doi: 10.4103/1735-3327.223613

30. Grover HS, Shukla S, Chug A, Singh K. Root coverage with buccal fat pad placed on restored cervical abrasion: Case report with three-year follow-up. J Indian Soc Periodontol. [Internet] 2018 [Consultado 2020 Jul 09];22(2):182-186. doi: 10.4103/jisp.jisp_312_17

31. Salehi-Nik N, Rezai Rad M, Kheiri L, Nazeman P, Nadjmi N, Khojasteh A. Buccal Fat Pad as a Potential Source of Stem Cells for Bone Regeneration: A Literature Review. Stem Cells Int. [Internet] 2017 [Consultado 2020 Jul 09];2017:8354640. doi: 10.1155/2017/8354640

32. Kablan F. The use of Buccal fat pad free graft in regenerative treatment of peri-implantitis: A new and predictable technique. Ann Maxillofac Surg. [Internet] 2015 [Consultado 2020 Jul 09];5(2):179-184. doi: 10.4103/22310746.175759

33. Guennal P, Guiol J. Use of buccal fat pads to prevent vestibular gingival recession of zygomatic implants. J Stomatol Oral Maxillofac Surg. [Internet] 2018 [Consultado 2020 Jul 09];119(2):161-163. doi: 10.1016/j.jormas.2017.10.017 